Décadrages Décadrages

cinéma, à travers champs Cinéma, à travers champs

19 | 2011

Autour d'Elephant de Gus Van Sant

\title{
Elephant ou les jeux vidéo en trompe-l'œil
}

\section{Selim Krichane}

\section{OpenEdition}

Journals

Édition électronique

URL : https://journals.openedition.org/decadrages/309

DOI : 10.4000/decadrages.309

ISSN : 2297-5977

\section{Éditeur}

Association Décadrages

\section{Édition imprimée}

Date de publication : 10 octobre 2011

Pagination : 70-84

ISBN : 978-2-9700668-3-5

ISSN : 2235-7823

Référence électronique

Selim Krichane, «Elephant ou les jeux vidéo en trompe-l'œil », Décadrages [En ligne], 19 | 2011, mis en ligne le 10 octobre 2012, consulté le 03 avril 2022. URL : http://journals.openedition.org/decadrages/ 309 ; DOI : https://doi.org/10.4000/decadrages.309 


\title{
Elephant ou les jeux vidéo en trompe-l'œil
}

\author{
par Selim Krichane
}

1 Mark Griffith, "Violent Video Games and Aggression: A Review of the Literature ", Aggression and Violent Behavior, vol. 4, $\mathrm{n}^{\circ} 2$, pp. 203212, 1999.

2 ld., p. 204.

3 Cette littérature n'a pas disparu au tournant du millénaire comme en témoigne le récent ouvrage de Lawrence Kutner et Cheryl K. Olson, Grand Theft Childhood: The Surprising Truth About Violent Video Games and What Parents Can Do (New York, Simon \& Schuster, 2008), vulgarisation d'une recherche mandatée par l'Etat américain (département de la Justice, bureau de la justice juvénile et de prévention de la délinquance).

4 Pierre Bruno, Les jeux vidéo, Paris, Syros (coll. "Ecole des parents"), 1993.

\section{Une peur fin de siècle (1990-2000)}

On a coutume d'associer le début du troisième millénaire à l'avènement des game studies, domaine d'études interdisciplinaires en devenir qui s'évertue à analyser les jeux vidéo dans leur fonctionnement interne, leurs implications sociales et idéologiques ou leurs rapports aux autres médias. Pourtant, déjà vingt ans plus tôt, un champ discursif dévolu aux jeux vidéo se dessine dans les universités. Les ouvrages et articles en question (émanant principalement des champs de la psychologie de la petite enfance ou comportementale) tentent de mesurer la dangerosité des objets vidéoludiques, leurs effets supposément néfastes sur le psychisme ou le comportement. L'analyse critique de cette littérature menée par Mark Griffith en 19991 - date du massacre de Columbine - relève le laxisme répandu en terme de recherche empirique ainsi que certaines lacunes méthodologiques systématiques. Ces recherches voient également s'affronter deux modèles contradictoires, d'une part la théorie de l'apprentissage social, d'autre part celle de la catharsis ${ }^{2}$. Ces deux prédicats gouvernent également les arguments que l'on trouve dans la presse ou encore dans les ouvrages généraux destinés aux parents inquiets ${ }^{3}$. Dans cette dernière catégorie s'inscrit parfaitement l'ouvrage de Pierre Bruno Les jeux vidéo, publié dans la collection "Ecole des parents» en 1993, qui, en réponse à la vague alarmiste, propose un discours plutôt nuancé sur son objet et fait figure de précurseur dans le champ des game studies, puisqu'il constitue une typologie des jeux vidéo (qui s'arrête aux «leaders» de l'époque, Sega et Nintendo) ainsi qu'une analyse idéologique de leur contenu 4 . La sortie d'Elephant (USA, 2003) s'inscrit dans le sillage de cette peur fin de siècle à l'endroit des jeux vidéo: alors que leur valeur scientifique et culturelle est négligée, l'accent est majoritairement mis sur la question de leur potentielle dangerosité. Le film de Gus Van Sant porte d'ailleurs, à notre sens, les stigmates de ces a priori contextuels.

Les discours alarmistes qui font florès dans les années 1990 seront souvent articulés, aux Etats-Unis, autour de la question des massacres 
dans les lycées vus comme autant de preuves irréfutables de l'effet néfaste des jeux sur la population adolescente. Ainsi, Dave Grossman n'hésitet-il pas, dans Stop Teaching Our Kids to Kill, à affirmer que les jeux vidéo permettent aux enfants d'apprendre à manier des armes à feu et attisent leurs instincts belliqueux ${ }^{5}$. Il débute son argumentaire par un recensement des tueries perpétrées dans les lycées américains. La peur à l'égard des jeux vidéo sera renforcée par ce type de massacres et relayée par un certain nombre de journalistes et d'experts. Ce débat public s'articule entre différentes sphères institutionnelles - journaux, universités, associations de parents, etc. - dont émanent des discours souvent partisans, fortement polarisés entre pro et contra. A l'autre extrémité du spectre se situe notamment le discours des frères Le Diberdier, qui suivent sans la nommer la théorie de la catharsis. Pour eux, la violence figurée dans les jeux vidéo (ainsi que dans les arts) sert d'exutoire aux joueurs, sans mener à des passages à l'acté 6 .

Si nous revenons sur ces discours formulés en faveur ou à l'encontre des jeux vidéo à la fin du siècle passé, c'est que nous estimons qu'Elephant n'est pas totalement détaché de ce regard sociétal qui a longtemps dominé l'opinion publique. Cette affirmation va a priori contre les postulats de la critique qui, reprenant en grande partie le discours du réalisateur, a vu en Elephant un film nuancé qui ne cherche pas à expliquer ni à démontrer les causes du massacre de Columbine, mais qui présente plutôt un éventail de facteurs, de pistes potentielles comme autant de "fragments d'un puzzle» $\mathbf{7}$. Si tant est que Gus Van Sant se "défie des réponses toutes faites $8 \boldsymbol{8}$ en proposant une structure énonciative polyphonique et une caractérisation rétrospective de certains personnages (comme le montre l'analyse d'Alain Boillat dans le présent numéro), toutes les "pistes" ne sont pas pourvues de la même pondération en fonction de leur durée, de leur mise en forme ou encore de leur articulation au sein du discours filmique. Il ne s'agit certes pas de faire d'Elephant un film manichéen, mais d'éclairer avec plus de précision, en termes d'emplacement syntagmatique ou encore de traitement visuel, la façon dont le fragment "jeu vidéo» est envisagé comme «cause potentielle du drame» 9 .

La connotation potentiellement péjorative des jeux vidéo dans Elephant est accompagnée d'un autre élément que nous aimerions développer, à savoir celui de l'intermédialité ou de l'hybridité du film et de son emploi de codes vidéoludiques. Il s'agit ici d'un trait plus général qui traverse le film et l'ensemble de l'œuvre de Gus Van Sant, comme le note Edouard Arnoldy dans son article. Avant de considérer cette intermédialité, il semble nécessaire de faire retour sur l'hybridité qui
5 Dave Grossman, Gloria DeGaetano, Stop Teaching Our Kids to Kill. A Call to Action Against TV, Movie and Video Game Violence, New York, Crown Publishers, 1999, pp. 2-5 et chapitre 4, pp. 65-81. Grâce au lieutenant-colonel Grossman, nous apprenons notamment que l'armée américaine s'est servie d'un simulateur de combat modélisé à partir de Doom (Marine Doom) à la fin des années 1990 (p. 77)!

6 Alain, Frédéric Le Diberder, Qui a peur des jeux vidéo?, Paris, La Découverte, 1993. Place importante que celle des frères Le Diberder dans l'histoire de la théorie du jeu vidéo, car ils clamaient l'"artisticité" de ce média en 1993, près de dix ans avant sa légitimation institutionnelle. Ils ont ensuite développé plus amplement leur propos dans L'Univers des jeux vidéo, Paris, La Découverte, 1998. La question de la dangerosité des jeux et de leur traitement médiatique y est à nouveau présente, pp. 89-91.

7 Philippe Rouyer, "Elephant: Marche funèbre", Positif, n 513, novembre 2003, p. 20.

$8 \mathrm{lbid}$.

9 Le cinéaste en fait mention dans l'interview incluse dans les bonus du DVD d'Elephant (About Elephant, Editions MK2) où il énonce les multiples pistes explicatives: le mauvais temps, les jeux vidéo, les insultes, etc. 
10 Alexis Blanchet, Des pixels à Hollywood. Cinéma et jeu vidéo, une histoire économique et culturelle, Paris, Editions Pix'n Love, p. 87.

11 Bolter et Grusin définissent la remédiation comme "la représentation d'un medium dans un autre " et la considèrent comme un "trait définitoire des nouveaux médias digitaux" [notre traduction]. Jay David Bolter, Richard Grusin, Remediation. Understanding New Media, Cambridge/Londres, MIT Press, 1999, p. 45.

12 L'utilisation de la 3D n'est pas chose nouvelle et remonte à la fin des années 1970 avec Tailgunner (Cinematronics, 1979) puis Battlezone (Atari, 1980) en 3D fil de fer ("wireframe $3 D ")$. La 3D polygonale amène des volumes pleins ainsi que des textures, parfois obtenues à partir d'images photographiques, comme dans le cas de Tomb Raider. La 3D polygonale se développe dans les années 1980 (citons I, Robot, 1983 de Dave Theurer pour Atari, Midwinter, de Mike Singelton en 1989) pour ensuite se généraliser dans les années 1990. Voir Tristan Donovan, Replay, the History of Video Games, Lewes, Yellow Ant, 2010, pp. 249-263.

13 Lorsque le tir est un mécanisme central d'un jeu "à la troisième personne ", on parle alors de TPS (Third Person Shooter). Alexis Blanchet en donne la définition suivante dans son lexique: "Genre vidéoludique désignant un jeu de tir en vue à la $3^{e}$ personne, (positionnée derrière le personnage à hauteur d'épaule) dans des environnements de jeu générés en $3 D$ ». Alexis Blanchet, op. cit., p. 446.

14 Picard, Martin, Pour une esthétique du cinéma transludique: figures du jeu vidéo et de l'animation dans le cinéma d'effets visuels du tournant du XXI siècle, thèse de doctorat sous la direction de Livia Monnet et Bernard Perron, Université de Montréal, 2009 (non publiée). Disponible en ligne: www.ludicine.ca/ [dernière consultation au 29.07.2011]. Des productions récentes comme Sucker Punch (Zack Snyder, USA, 2011) ou Scott Pilgrim vs. the World (Edgar Wright, USA, 2010) s'inscrivent également dans ce paradigme.

$15 / d .$, p. 80. Picard emprunte ses catégories de remédiatisation à Sandy Baczkowski, La contamination du cinéma américain contemporain par les jeux vidéo: convergences et divergences. Thèse de doctorat, Université de Toulouse II-Le Mirail, 2005, p. 56 (cité par Martin Picard). marque l'histoire des jeux vidéo, afin de mieux articuler ces échanges médiatiques.

\section{Hybridité d'une histoire, histoire d'une hybridité}

L'histoire des jeux vidéo est marquée par des emprunts constants au cinéma, aussi bien en termes de structuration du narratif que de facture visuelle. L'ouvrage d'Alexis Blanchet montre combien l'histoire économique et culturelle des médias a rapproché les jeux vidéo du cinéma, avec notamment l'exemple emblématique de l'achat d'Atari - alors leader incontesté des jeux vidéo - par Warner Communications en 197610. S’ensuit une longue chaîne de procédés de "remédiation» (remediation) $\mathbf{1 1}$ avec notamment deux étapes qui nous semblent ici centrales : l'accroissement du photoréalisme dans les années 1990 par l'utilisation de textures (parfois photographiques) et la généralisation de la « $3 \mathrm{D}$ polygonale \$12 accompagnée de perspectives mathématiques qui miment les perspectives géométriques et photographiques. Se développent aussi dans les mêmes années les jeux dits «à la première personne», en vue subjective, ou «à la troisième personne»13, en vue semi-subjective. Les premiers seront popularisés par les fameuses productions d'id Software (Castle Wolfenstein 3D, Doom, Quake). Les seconds susciteront un engouement similaire quelques années plus tard, avec notamment Lara Croft (Core Design, 1996) ou Syphon Filter (Eidetic, 1999). Les jeux à la première personne susmentionnés sont ceux auxquels jouaient les deux adolescents de Columbine et, nous le verrons plus en détails, ceux qui sont partiellement cités par le film. En outre, un grand nombre de procédés filmiques récurrents employés dans Elephant font écho aux jeux «à la troisième personne» (fig. 1-2).

L'intermédialité mise en œuvre dans Elephant repose donc en partie sur les procédés de remédiation propres à l'histoire des jeux vidéo qui les rapprochent du cinéma dans le courant des années 1990. Suite à cette complicité médiatique se dessine un paradigme cinématographique de remédiation inverse par lequel les jeux vidéo marquent à leur tour certaines productions filmiques. Dès lors, Elephant revêt un caractère "transludique», selon l'appellation de Martin Picard, en s'appropriant une série de procédés vidéoludiques $\mathbf{1 4}$. L'analyse de Picard s’arrête au cinéma "d'effets visuels» (Matrix, Avalon), mais il nous semble possible d'élargir la notion à un film comme Elephant. Picard mentionne d'ailleurs brièvement le film en parlant de «remédiatisation esthétique»15. Si le terme «esthétique» est quelque peu flou et désigne insuffisamment la particularité d'Elephant, il faudrait ajouter que celui-ci s'inscrit dans un contexte économique et institutionnel différent des films qui intéressent 
Picard. Dans le champ du cinéma expérimental (ou «d'art et d'essai»), les correspondances avec les jeux vidéo sont autres que dans Matrix ou Avalon. Aux emprunts directs - sans ambiguïté - s'oppose, dans Elephant, un détournement de procédés vidéoludiques, notamment par la mise en cadre de chaque personnage focal ou par le traitement de temporalités que nous qualifierons d'algorithmiques.

\section{Personnages-pivots}

Un des traits définitoires des jeux en vue subjective tient à la systématicité qu'ils instaurent entre le point de vue et le personnage joué - l'«avatar». Dans le cas des FPS (First Person Shooters), la vue subjective devient un pivot de l'action, marqué par l'arme de l'avatar en amorce:

«La caméra à la première personne des FPS n'est pas uniquement une caméra, mais une caméra et une arme à feu jointes dans le même dispositif virtuel, une caméra-arme à feu. Ce dispositif ne permet pas à l'arme d'être déplacée indépendamment de la caméra qui est elle aussi chevillée au corps quasiment invisible du joueuravatar. Lorsque l'arme bouge, le cadre de la caméra ainsi que le corps de l'avatar l'accompagnent, et vice versa.»16

Le point de vue dans les jeux en question est souvent appelé "caméra» - comme dans la citation de Klevjer - aussi bien par les théoriciens que par les journalistes ou les joueurs. Bien que trop rarement problématisé, cet emploi métaphorique du terme «caméra» s'explique par la parenté

16 Rune Klevjer, "The Way of the Gun. The aesthetic of the single-player First Person Shooter ", p. 2 [notre traduction]. Disponible en ligne sur le site du Rune Klevjer: http://folk.uib.no/ smkrk/ [dernière consultation au 29.07.2011]. Publié dans Matteo Bittanti, Sue Morris (éd.), Doom. Giocare in prima persona, Milan, Costa \& Nolan, 2006.

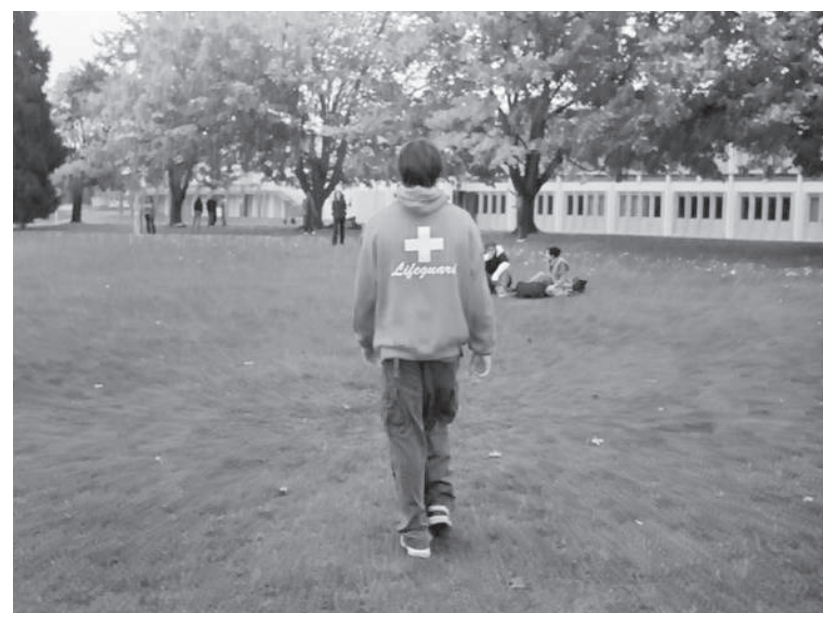

1

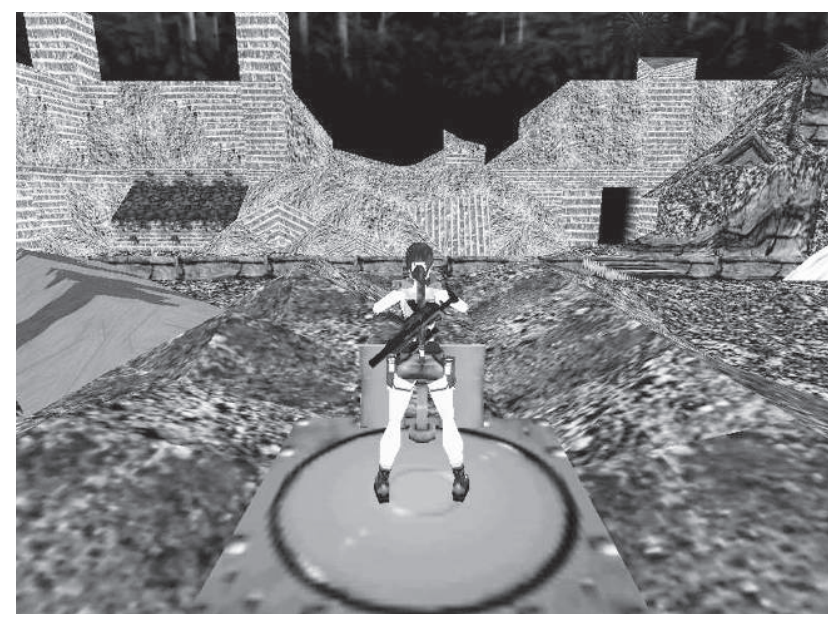

2 
17 Mario Bros. (Nintendo, 1983).

18 Christian Metz, Langage et cinéma, Paris, Editions Albatros, 1977 [1971], p. 46. On emploiera sans complexe la notion de code pourtant critiquée depuis son âge d'or dans les années 1970, mais uniquement pour renvoyer aux codes strictes, algorithmiques de la visualisation vidéoludique. Ceux-ci se rapprochent en outre, par leur systématicité, des "codes technologiques" chez Metz (id., p. 144).

19 Britta Neitzel, "Point of View and Point of Action. Eine Perspektive auf die Perspektive in Computerspielen", [2007], cité par Benjamin Bell dans First Person Perspectives. Point of View und Figurenzentrierte Erzälformen im Film und im Computerspiel, Münster, Lit Verlag Münster, 2010, p. 64.

20 Doom (id Software, 1993) n'est d'ailleurs pas en 3D, mais simule le déplacement dans les couloirs par la successivité de vues bidimensionnelles. Voir Rune Klevjer, op. cit., note 2. qu'entretient le calcul de la visualisation dans les jeux en vue subjective avec le dispositif cinématographique. Dans les deux cas, un agent au sein d'un espace vient "capter» un rendu visuel (captation lumineuse d'une part, simulation d'une perspective par un calcul d'autre part). Le point de vue dans ces jeux est mobile et en rapport direct avec un espace construit en $3 \mathrm{D}$, potentiellement visible sous mille coutures. Contrairement à un Mario $\mathbf{1 7}$ traditionnel qui se constitue en "bandes" horizontales successives (on parle alors de «side-scrollers»), les jeux en question instaurent un rapport variable entre l'espace et sa visualisation. L'idée d'un dispositif virtuel qui allie caméra et arme à feu de manière systématique souligne l'importance, dans les FPS, de ce que nous pourrions appeler les "codes généraux». Dans Langage et cinéma, Metz définit les "codes cinématographiques généraux» comme des «instances systématiques au compte desquelles [sont] versés les traits qui, non seulement caractérisent en propre le grand écran, mais de surcroît sont communs à tous les films»18. Le caractère informatique des jeux vidéo renforçant la systématicité du rendu visuel, la présence de tels codes ("codes vidéoludiques généraux", pourrions-nous dire) dont la conceptualisation est libérée du caractère métaphorique qu'imposaient la linguistique et la sémiologie est très répandue dans l'ensemble des objets vidéoludiques. Contrairement au point de vue cinématographique qui varie au gré des pratiques de montage selon des combinaisons quasiment infinies, la visualisation vidéoludique est en effet régie par des codes algorithmiques que l'on peut souvent dégager par l'analyse puisqu'ils découlent, en dernière instance, d'une programmation informatique, d'une séquence binaire réalisée afin de générer ces systématicités visuelles. Celles-ci permettent en outre de faciliter l'interactivité : possibilité de lire l'image, de s'y adapter et d'activer les différents mécanismes de jeu. Au point de vue s'ajoute donc le "point of action", selon la dénomination de Britta Neitzel qui correspond à «la position au sein de l'univers de jeu à partir de laquelle les actions sont accomplies»19.

Historiquement, les processus de visualisation dans les jeux en vue subjective sont marqués par un accroissement progressif des possibilités de déplacement et de manipulation du point de vue par le joueur. Dans les premières productions du genre chez id Software, le joueur pouvait uniquement se déplacer au sein de l'espace représenté sur un plan bidimensionnel20 : effectuer un mouvement de pivot, avancer, reculer. Avec Quake (id Software, 1996) ou System Shock (Looking Glass Studios, 1994) s'ajoute la possibilité d'effectuer des mouvements verticaux : regarder vers le bas ou le haut. On note une évolution similaire dans les jeux à la troisième personne: Lara Croft n'offrait que la possibilité de dépla- 
cer le personnage au sein de l'espace, alors qu'à partir du cinquième opus, l'axe de la caméra devient mobile indépendamment du personnage. Cette division entre mouvement de la caméra et mouvement du personnage joué fait aujourd'hui école dans les productions de ce type. Dans World of Warcraft (Blizzard Entertainment, 2004), Assassin's Creed (Ubisoft, 2007) ou encore Uncharted (Naughty Dog, 2007), pour ne citer que quelques exemples, le joueur a la possibilité de faire pivoter la caméra tout autour du personnage, de sorte à pouvoir choisir l'angle le plus «lisible» à n'importe quel moment du jeu, ou encore à pointer la "caméra"sur son avatar, le transformant alors momentanément en objet du regard à valeur attractionnelle $\mathbf{2 1}$.

On voit à quel point l'esthétique d'Elephant fait appel à un code vidéoludique de représentation et systématise une pratique de mise en cadre qui fait écho aux jeux "à la troisième personne». Les travellings avant dans les couloirs du lycée reproduisent la symétrie mise en œuvre dans ces jeux entre personnages joués (dans le film, nous dirions "personnages filmés») et point de vue. Le cadrage des acteurs en amorce, bien que l'échelle de plan soit variable, reste constant lors de leurs déambulations, et l'on voit de manière systématique se profiler le cadrage des «virages» qu'ils amorcent dans les couloirs: léger travelling latéral accompagné d'un mouvement panoramique qui dévoile les visages de trois quarts, puis retour à une position de dos (ou frontale). Mais la caméra d'Elephant n'est pas l'algorithme de calcul du rendu visuel d'un jeu en vue semi-subjective, elle se permet des errances que ce dernier ne connait pas 22.

Si le terme de "vue semi-subjective" est souvent repris dans le contexte des études vidéoludiques pour désigner les jeux à la troisième personne, il est frappant de noter à quel point la «subjectivité» des travellings avant en plan-séquence d'Elephant est malmenée. Avec Mitry, qui propose le terme dans Esthétique et psychologie du cinéma, on peut affirmer que le personnage en amorce "occupe une situation privilégiée dans le cadre» ou encore que "la caméra l'accompagne dans ses déplacements", mais sans pour autant qu'elle ne "voi[e] comme lui et en même temps que lui»23. L'absence de profondeur de champ renverse la lisibilité de l'image semi-subjective: plutôt que de valoriser ce qui est vu par le personnage, c'est le personnage lui-même qui est mis en exergue. Vue "semi-semi-subjective"? De même, bien que l'on soit tenté de penser à l'identification secondaire chez Metz qui désigne une identification au regard d'un personnage $\mathbf{2 4}$, il semble que le choix de la focale tend à amoindrir celle-ci au profit de l'identification primaire à la caméra. Les mouvements de pivot qui nous font passer du dos des personnages à une
21 Pratique courante dans les jeux de rôles où l'évolution du personnage est fonction de son accoutrement, des objets en sa possession, etc. Il est alors fréquent que le joueur contemple son personnage qui, par son apparence, dénote la progression du joueur. Autre dynamique de regard dans les jeux avec des personnages féminins, qui comme Lara Croft sont construits par les codes vidéoludiques comme objets du regard - et du désir - masculin. Ainsi la possibilité de faire le poirier dans Lara Croft n'a-t-elle aucune autre fonction sinon celle de constituer une plus-value monstrative.

22 Voir par exemple la séquence au comptoir de la cantine, discutée dans l'article d'Alain Boillat (fig. 9-12).

23 Jean Mitry, Esthétique et psychologie du cinéma, vol. 2, Paris, Editions Universitaires, 1965, pp. 77-78.

24 Christian Metz, Le signifiant imaginaire, Paris, Christian Bourgois, 2002, pp. 70-79 [Union Générale d'Editions, 1977]. 
25 François Jost, L'œil-caméra, Lyon, Presses Universitaires de Lyon, 1989 [1987], p. 26.

26 C'est aussi et surtout l'aveuglement des deux jeunes tueurs qui se concrétise dans la séquence de la bibliothèque où la tuerie est étagée dans la profondeur du champ. Tandis que l'avant-plan est occupé par les corps et les armes des tueurs, l'acte est rejeté à l'arrièreplan, demeurant flou.

27 Edward Branigan, Point of View in the Cinema, Berlin/New York/Amsterdam, Mouton Publishers, 1984, pp. 132-138 et pp. 123-126.

28 Jean Mitry, op. cit., p. 78. vue frontale fragilisent eux aussi la «subjectivité» des plans-séquences en les faisant définitivement passer du côté d'une "ocularisation zéro» $\mathbf{2 5}$. Si l'emploi généralisé du flou tend à diminuer le caractère subjectif de l'ocularisation mise en ouvre par les travellings d'Elephant, il ne faut pas pour autant occulter son caractère psychologisant et donc potentiellement subjectivisant. Le flou des travellings renvoie certainement à l'impuissance des personnages, à leur aveuglement et préfigure ainsi la rupture qu'instaure le film entre les déambulations du quotidien et le massacre constamment «repoussé» par le discours filmique 26.

Dans son ouvrage sur le point de vue au cinéma, Branigan distingue les vues subjectives "classiques» de celles qui constituent une référence psychologique à un état intérieur - point de vue qu'il nomme «projectif»27. Parallèlement, on trouve chez Mitry une catégorie à laquelle on pourrait rapporter les plans-séquences d'Elephant. Il s'agit de «l'image totale» qu'il construit comme sous-catégorie de "l'image semi-subjective»:

«A la limite, le personnage en question [en amorce, dans l'image semi-subjective] peut servir de truchement, de telle sorte que son point de vue coïncide avec celui de l'auteur. On obtient alors ce que nous appellerons une image totale, c'est à dire à la fois descriptive (par ce qu'elle montre), analytique (identifiée à la vision du personnage) et symbolique (par les structures compositionnelles qui en découlent). $\mathbf{2 8}$

En prenant la liberté d'appliquer le vocable de Mitry au film en question, on remarque qu'un rapport de proportionnalité inverse se dessine entre les dimensions "descriptive» et «symbolique» des plans-séquences. En d'autres termes, l'effacement du champ de vision des personnages focalisés amène à la coloration «symbolique» des plans. En parallèle, le caractère "descriptif» des plans est subordonné au marquage analytique: le spectateur sait qu'il "voit avec" les personnages d'Elephant, bien qu'il ne partage que rarement leur champ de vision. C'est précisément dans cette négation de la vision subjective que s'opère le détournement du code vidéoludique. Malgré un effet de citation fort, le trait saillant de l'économie visuelle des jeux vidéo, c'est-à-dire la lisibilité de l'image, est absent. Cette absence - qui rendrait tout jeu proprement injouable revêt dans le film, pour paraphraser une dernière fois Mitry, une valeur «symbolique».

\section{Temporalités: rapports algorithmiques}

Bien que l'on ne voie pas "avec" les personnages d'Elephant, il est certain que l'on est amené à voir «en même temps » qu'eux leur quotidien 
de lycéens. Cette continuité temporelle est particulièrement palpable dans les plans-séquences, mais aussi dans les ralentis et accélérés qui ponctuent le métrage. Dans les deux cas, nous sommes face à un rapport algorithmique entre le temps de l'histoire et celui du récit. Dans le premier cas, un rapport d'équivalence détermine le régime temporel, et, dans le second, un rapport continu de dilatation (ou de condensation). Il se trouve que ces régimes temporels sont dominants dans le champ des jeux vidéo dans lequel l'interactivité appelle une continuité du flux audiovisuel. Cette particularité des jeux vidéo a conduit grand nombre de théoriciens des game studies à affirmer que la narratologie n'était pas adaptée à leur objet ${ }^{29}$.

Ainsi Markku Eskelinen affirme que l'absence de discours sur le passé dans l'activité vidéoludique la place en dehors du champ de la narration, caractérisé par l'absence d'une double temporalité ${ }^{\mathbf{3 0}}$. La simulation du jeu impliquerait donc une représentation où la seule temporalité nécessaire serait celle du déroulement du jeu «en flux continu» et de l'interaction avec le joueur, calquée sur la temporalité du jeu. Kevin Shut, dans son article sur la narrativité dans les jeux vidéo, reprend l'argument de Jesper Juul31 selon lequel les jeux vidéo ne peuvent pas manipuler la temporalité à la manière des romans ou des films du fait de l'interactivité qui s'opère en temps réel. Ainsi note-t-il que, dans Europa Universalis (Paradox Interactive, 2000), il n'y a "pas de distinction entre temps extérieur et temps intérieur»32. Europa Universalis est un jeu de stratégie en temps réel (par opposition aux jeux en "tour par tour» [turned-based]) dans lequel l'interface présente une vue aérienne d'un vaste terrain où le joueur va déployer des forces, construire des bâtiments, rassembler des ressources, etc. ${ }^{33}$. Mais lorsqu'il parle de temps réel et d'absence de distinction entre le temps du récit et le temps de l'histoire, Kevin Shut oublie que le cadre historique parcouru dans le jeu s'étend sur environ trois cent ans. En effet, une seconde dans l'expérience du jeu correspond à plusieurs heures dans l'épopée historique des jeux de stratégie, selon un rapport constant. De même, l'animation de la construction d'un bâtiment, dans ce type de jeu, est souvent "accélérée». Cette temporalité n'est pas unique et coexiste bien avec des représentations en temps réel: les personnages se déplacent et combattent en temps réel (l'accélération vertigineuse du temps du récit ne les affecte pas). Plusieurs temporalités sont donc à même de coexister au sein d'un jeu. C'est précisément ce rapport algorithmique, cette cadence automatisée entre deux temporalités qui constitue la spécificité de certains jeux vidéo à laquelle le traitement temporel d'Elephant fait écho.
29 Pour un compte rendu du débat narratologie/ludologie, voir Gonzalo Frasca, "Ludologists Love Stories, Too: Notes from a Debate that Never Took Place", in Marinka Copier, Joost Raessens (éd.), Level Up: Digital Games Research Conference Proceedings, Utrecht, DiGRA/University of Utrecht, 2003. Disponible en ligne: www.digra.org/dl/db/05163.01125 [dernière consultation au 29.07.2011].

30 Markku Eskelinen, "The Gaming Situation", Game Studies: the International Journal of Computer Game Research, vol. 1, $\mathrm{n}^{\circ}$ 1, 2001, pp. 2-3. Eskelinen cite Metz pour parler de la double temporalité du récit.

31 Jesper Juul, "Games Telling Stories? A Brief Note on Games and Narratives ", Game Studies: the International Journal of Computer Game Research, vol. 1, $\mathrm{n}^{\circ}$ 1, 2001, p. 7, paragraphe intitulé "La temporalité dans les jeux vidéo".

32 Kevin Shut, "Technology tells a tale: digital games and narrative", Game Studies: the International Journal of Computer Game Research, vol. 1, n 5, 2004, p. 7.

33 Kevin Shut dit à propos de ce jeu: "Il s'agit d'un jeu en temps réel (c'est-à-dire que le temps du jeu est continu) de simulation historique qui permet au joueur de contrôler et de vivre l'aventure d'une nation dans la lutte géopolitique européenne de conquête mondiale et de monopole économique durant la période 1492-1789.", id., p. 6 [notre traduction]. 
34 [Notre traduction]. Gerry va ensuite décrire sa conquête de Thèbes par la présentation d'une série d'événements propres aux jeux de stratégie, parodiant ainsi les mécanismes de jeu du genre: destruction d'un temple par un accident naturel, impossibilité de le reconstruire par manque de ressources, gestion des "unités " d'une armée, etc.
La référence faite aux jeux vidéo dans le film précédent de Gus Van Sant, Gerry (USA, 2002), achèvera de nous convaincre de la pertinence du rapprochement effectué ici entre le régime de temporalité d'Elephant et les codes vidéoludiques. Lors de la première nuit passée dans le désert, au coin d'un feu, Casey Affleck narre une session de jeu à Matt Damon. Il s'agit en l'occurrence d'un jeu de stratégie similaire à celui utilisé par Shut dans son argumentaire anti-narratologique. Et c'est justement la temporalité accélérée du jeu à laquelle les dialogues font référence:

"Casey Affleck: - J'ai conquis Thèbes.

Matt Damon:- Quand?

Casey Affleck: - Il y a deux semaines. [...] J'ai dit à Gerry: 〈J'ai régné sur ce territoire pendant quatre-vingt-dix-sept ans et maintenant je veux le récupérer ».»34

L'effet de contraste obtenu par cette référence vidéoludique est bien évidemment renforcé et motivé par le cadre narratif épuré du film (et de la scène). Le dialogue convoque par ailleurs un état antérieur du temps de l'histoire filmique ("il y a deux semaines») et accélère par le compte rendu oral la temporalité déjà précipitée du temps du jeu. La temporalité continue des plans-séquences d'Elephant rappelle ces jeux «en temps réel» et produit, comme le défilement des couloirs dans les jeux de tir à la première personne, un effet envoûtant. A cela s'ajoutent les points de rupture des accélérés et ralentis. Les vues du ciel accélérées en contreplongée instaurent un rapport épique entre le récit et les sphères cosmiques, à la manière des jeux de stratégie en temps réel. Les ralentis,

Les ralentis d'Elephant (USA, 2003): spectacularisation des corps et emphase des regards.

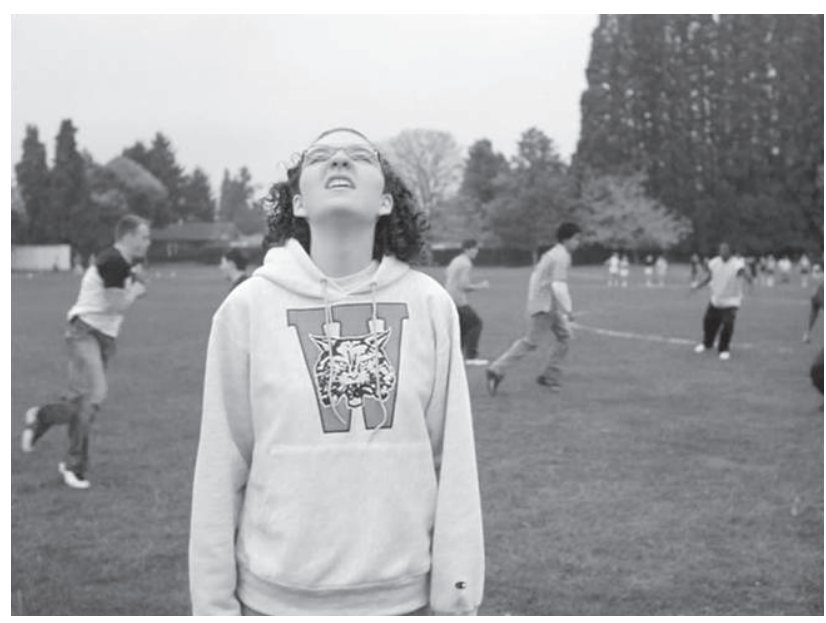

3

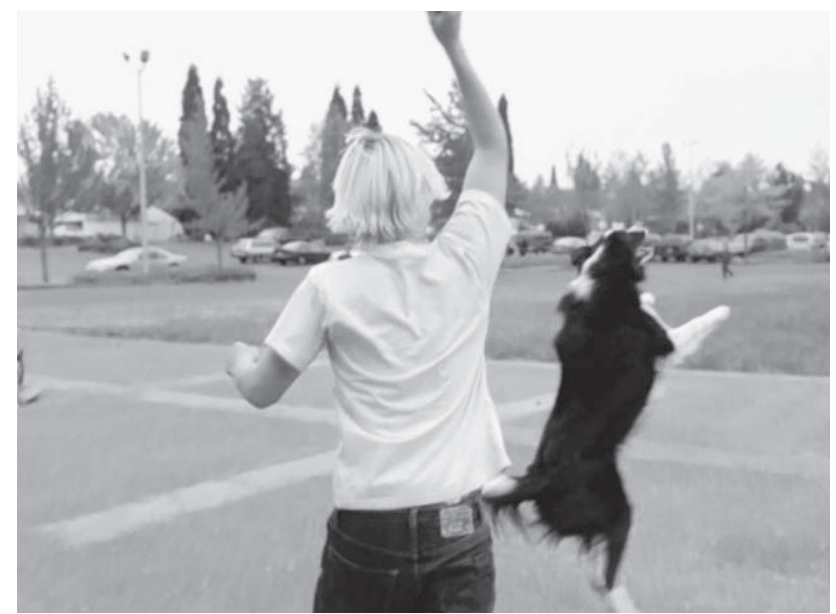

4 
quant à eux, recouvrent au moins deux fonctions distinctes. D'une part, ils pondèrent le film d'effets attractionnels par la dilatation de mouvements spectaculaires (l'entraînement de football américain au début du film, puis le saut du chien lorsque John quitte le lycée, fig. 3-4) et viennent souligner des regards, parfois diffus (Michelle sur le terrain de sport) ou ciblés (Brittanny dévisageant Nathan, avant sa rencontre avec Carrie). L'effet attractionnel de ce procédé (couplé dans le film à un effet d'emphase des regards et des déplacements) est également présent dans certains jeux vidéo, comme Max Payne (Remedy Entertainement, 2001), où il permet (au-delà de sa fonction d'aide au joueur lors de séquences difficiles) de spectaculariser les scènes d'action.

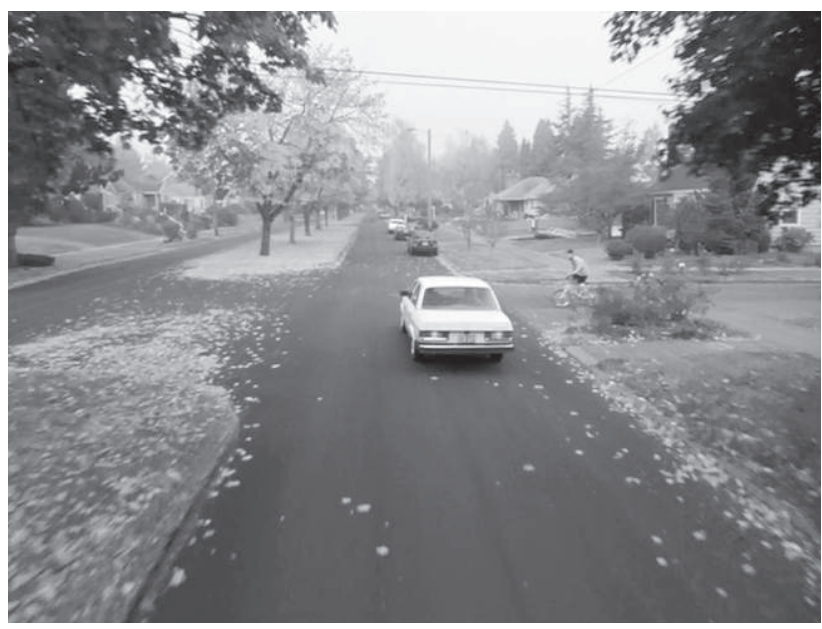

\section{Du film transludique au jeu dans le film}

Aux travellings des plans-séquences qui ponctuent le film viennent s'ajouter deux plans qui sont plus proches des jeux vidéo en reproduisant un flux visuel qui pourrait être celui d'un jeu. Le plan sur la voiture lors de la séquence d'ouverture (fig. 5) correspond à peu de chose près à une possibilité de visualisation d'un jeu tel que Grand Theft Auto III (Rockstar Games, 2001). Le second apparaît lors de la séquence de planification de l'attaque qui est ponctuée par des plans d'inserts (brèves prolepses) et reproduit la représentation typique des FPS dont nous avons fait mention plus haut (dispositif "caméra-arme à feu " de Klevjer, fig. 6-7). Le plan sur la voiture fait écho à un plan similaire en ouverture de Gerry, bien que celui-ci soit plus proche d'une vue semi-subjective (fig. 5/8-9) 35. Dans Elephant cependant, la conduite chaotique du père
5/ Filature automobile en ouverture d'Elephant.

35 Contrairement au travelling en plongée dans Elephant, le plan de Gerry est filmé à hauteur des personnages dans la voiture et se rapproche progressivement du point de vue des personnages. Notons que le motif de l'automobile filmée à distance depuis derrière constitue la clôture du film Will Hunting (Gus Van Sant, USA, 1997). 
36 A la liste des supports diégétiques ajoutons les partitions, les pages internet consultées ainsi que le livre lu par Alex. de John accentue le parallèle avec Grand Theft Auto dans lequel le code de la route est rarement respecté. Le risque d'accident ou l'anticipation suscitée par l'apparition d'un cycliste dans le champ accentue la propension de la part du spectateur à l'identification au conducteur. Ces exemples de cas limites ou extrêmes indiquent que l'hybridité médiatique ou la nature de la remédiation cinéma/jeu vidéo n'est pas homogène dans Elephant. A cet égard, le mode dominant de représentation demeure celui des travellings avant dans les couloirs du lycée, bien qu'ils soient comme balisés dans la temporalité du récit par des plans qui font figure de remédiation maximale.

La séquence qui thématise les jeux vidéo dans le film se détache particulièrement du reste par les mouvements de caméra qui la traversent, ainsi que par le foisonnement d'écrans, de cadres dans le cadre. La séquence en question dépeint les deux adolescents dans la chambre d'Eric, l'un jouant au piano et l'autre à un FPS. Après l'interlude musical, l'ordinateur sert à l'achat d'une arme automatique. Puis, le lendemain, les adolescents reçoivent, devant un documentaire sur le nazisme, l'arme à feu achetée la veille sur internet. Il est frappant de noter la surcharge d'écrans et de médias mis en scène: écran d'ordinateur, télévision, fenêtre de la chambre par laquelle est indiquée l'arrivée d'Alex, embrasure de la porte, puis paroi vitrée derrière la TV qui permet de montrer simultanément la télévision en "nature morte» et l'arrivée du

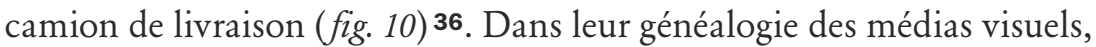
Bolter et Grusin désignent l'hypermédialité comme l'un des traits carac-

Le film mime la vue subjective des FPS et le dispositif "caméra-arme à feu ".

Doom (id Software, 1993). @ id Software.
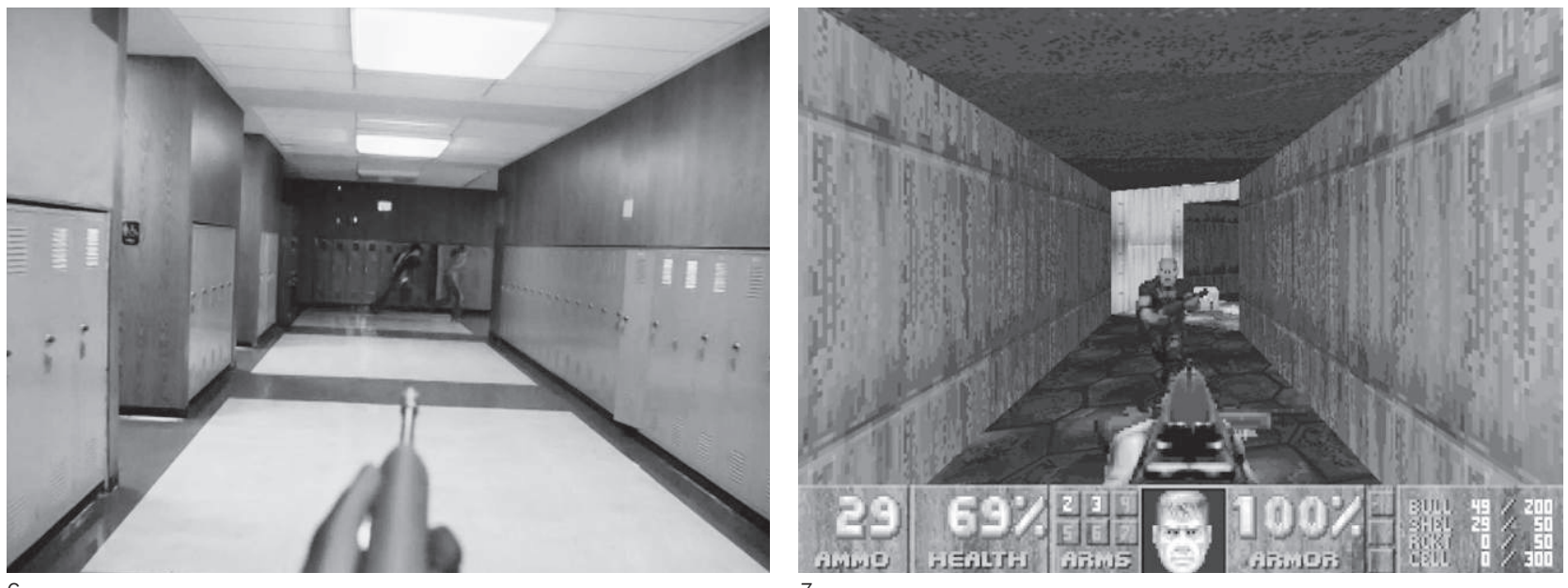

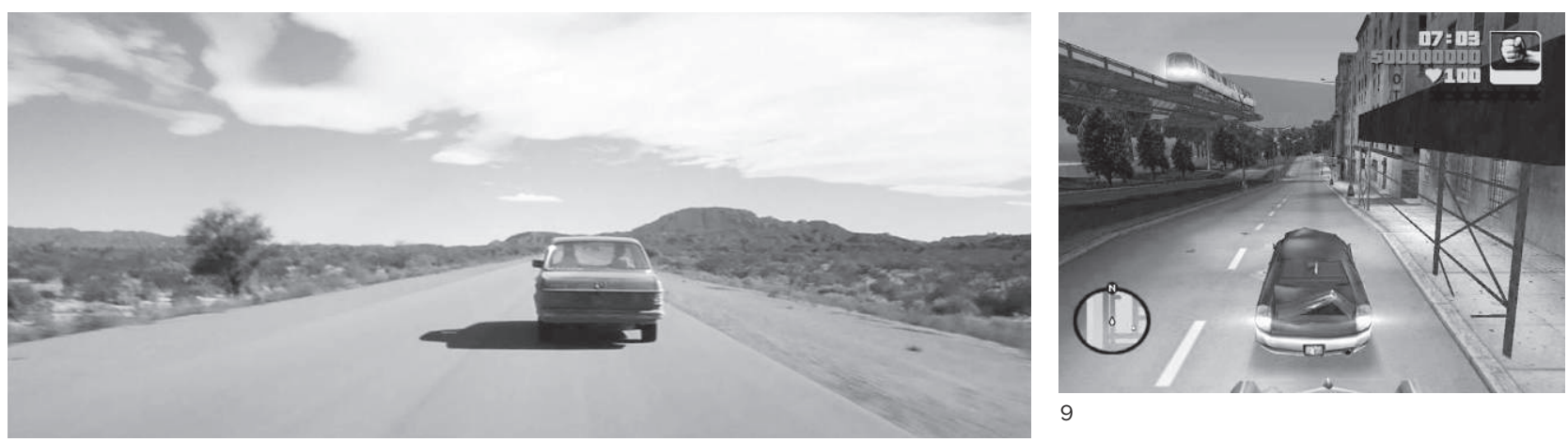

L'ouverture de Gerry (USA, 2002) et un trajet en automobile dans Grand Theft Auto III (abrégé GTA III, Rockstar North, 2001). @ Rockstar Games.

téristiques des échanges intermédiaux. La multiplicité des écrans et des supports 37 , à laquelle le cinéma se prête particulièrement bien du fait de la pluralité des matières de l'expression qu'il engage, se déploie de 37 Jay David Bolter, Richard Grusin, op. cit., manière particulièrement sensible dans cette séquence. D’une part, la stratégie de représentation permet de dire la multiplicité des médias comme élément constitutif de la vie des adolescents - discours à valeur généralisante/documentarisante - ainsi que les rapports différentiels entretenus par les personnages avec ceux-ci (affinité pour les jeux vidéo et méconnaissance des documents d'archives relatifs au nazisme) afin de suggérer un enchaînement causal qui mène potentiellement du jeu de tir à l'obtention d'une arme à feu, en passant par un site de vente

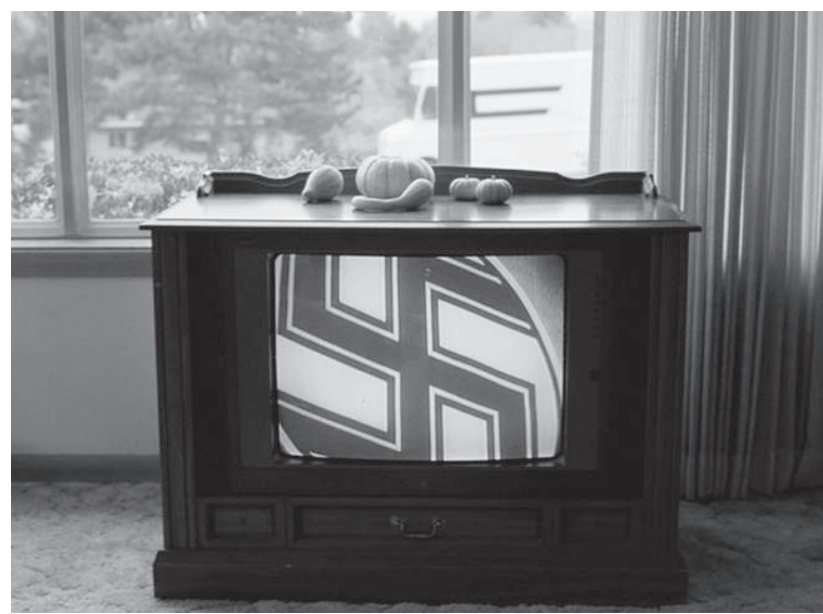

10/ Multiplicité des tableaux au sein du cadre. L'arme à feu commandée sur internet est apportée par un camion de livraison situé à l'arrière-plan. 
38 Notons que ce film dont Alain Boillat explique le rôle de référence qu'il a joué pour Gus Van Sant est antérieur aux jeux que nous citons dans le présent article. Mais les jeux en vue subjective se développent dès le début de l'histoire du médium et nous ne visons pas à établir de lien d'influence ou de causalité direct, mais bien plutôt une généalogie non linéaire des rapports intermédiaux dans laquelle s'inscrit également le film de Clarke.

39 Aspect similaire, sinon renforcé, dans Gerry. La mécanicité des mouvements y étant motivée diégétiquement par l'état de fatigue des personnages.

40 En ce qui concerne la façon dont Gus Van Sant a fait concevoir ce jeu sur la base de son film Gerry, et les questions que ce choix soulève en termes de remake et de remédiation, voir l'article d'Alain Boillat dans le présent dossier.

41 Encadré dans le coin inférieur gauche de l'image avec les visages en vue frontale des protagonistes: ici Casey Affleck et Matt Damon ainsi que le "Gerrycount" qui parodie (selon la logique Schtroumpfesque de Gerry) le "fragcount " des FPS; notion elle aussi instituée par Doom et qui renvoie au score du joueur, c'està-dire au nombre de cibles abattues.

42 Ce procédé de différenciation, au-delà de sa valeur psychologisante associée à la vue subjective (les jeux vidéo comme échappatoire, opposés à l'enfermement et au tournis de la chambre à coucher) résonne avec le "système" Gerry-Elephant. Différence nette entre les espaces cloisonnés d'Elephant et les grands vides désértiques de Gerry. L'espace ouvert à l'infini n'en est pas pour autant moins labyrinthique.

43 Voir Rune Klevjer, op. cit., p. 2 [notre traduction]. d'armes. Le panoramique répété et répétitif illustre (en faisant deux fois et demie le tour de la pièce) l'espace de "vie» des adolescents suivant une rythmique marquée entre déplacements des personnages, mesures musicales et mouvements de caméra. A l'instar de la séquence au piano dans Week-end de Jean-Luc Godard (France, 1967), la musique diégétique alterne entre son in et off à mesure que la caméra pivote, présentant l'espace de manière itérative, comme entraînée par les portées musicales. Ce caractère mécanique des mouvements est relativement continu au sein du film et suscite un lien supplémentaire entre le film et les jeux vidéo, encore plus palpable dans le film originel de Clarke (Elephant, $\mathrm{GB}, 1989)^{38}$, où la répétitivité et la mécanicité sont portées à leur apogée. On a de surcroît affaire à des mécanismes de jeux courants : "déplacement», «tir», "course» 39.

Le jeu fictif représenté dans le film de Gus Van Sant40 dans une vue subjective (plan qui se démarque puisqu'il n'y en a que très peu dans le film), construit plusieurs liens intertextuels sur lesquels nous allons nous arrêter. Proche des FPS par le mime de l'interface de jeu $\mathbf{4 1}$ et le "dispositif caméra-arme à feu ", le jeu vidéo d'Elephant s'en démarque néanmoins par l'abandon de «l'espace tunnel» (présent dans les plans-séquences du lycée) caractéristique de la première génération de FPS 42 au profit de l'espace désertique de Gerry (fig. 11). L'espace tunnel renvoie aux réseaux de couloirs et de portes qui foisonnent dans les jeux de tirs à la première personne. Bien qu'un tel agencement de l'espace ludique soit initialement dû aux restrictions techniques de l'époque, selon Klevjer, la "perspective réduite augmente la sensation de vitesse, mais aussi l'impression de désorientation et de suspense»43. On retrouve la même désorientation accentuée par les flous dans les plans-séquences d'Elephant.

Finalement, l'élément central du jeu vidéo fictif tient dans les mouvements de caméra et l'interaction avatar/victimes qui ne correspondent en rien aux FPS, mais singent les mouvements mis en œuvre dans les travellings en plan-séquence d'Elephant. Convocation d'une part des codifications visuelles d'un genre vidéoludique et d'autre part d'éléments narratifs et de procédés filmiques des propres films expérimentaux de Van Sant. Par cette caractérisation particulière - détournée - d'un genre vidéoludique, Van Sant fait du jeu vidéo un objet plus radical qu'il ne l'est en réalité, souscrivant alors aux commentaires de la presse en période de controverses alarmistes. Dans son article paru dans le numéro spécial du Times du 24 avril 1999 dédié au massacre de Columbine et aux enregistrements vidéo ( $b$ basement tapes») qu'ont réalisés les adolescents avant la tuerie, Amy Harmon mentionne les jeux vidéo auxquels jouaient Harris et Klebold «dans le[s]quel[s] le joueur traque [stalks] et tue ses 


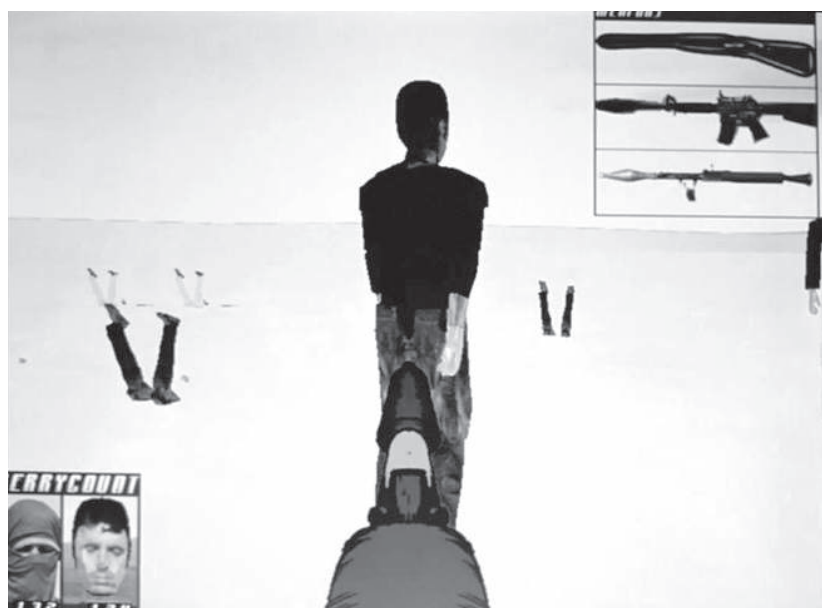

adversaires» 44. Dans les deux cas apparaît l'idée d'une confrontation de dos qui suppose un joueur-tueur, alors que les FPS se déroulent globalement en confrontations frontales entre joueur-avatar et adversaires 45 . Ajoutons encore le rapport suggéré dans la séquence entre jeux vidéo et achat d'arme sur internet auquel le discours filmique réserve un traitement similaire (vue subjective, cadrage plein sur l'écran d'ordinateur) et une proximité syntagmatique qui, par la contiguité, semble suggérer une certaine forme de continuité, voire un développement causal46. Les transcriptions des «basements tapes» vont aussi provoquer la circulation de "citations" des tueurs. La presse internationale diffusera les paroles de Harris qui dit de son arme à feu qu'elle «sort tout droit [du jeu vidéo] Doom»47. Etonnant de voir dans un film qui en apparence fait fi du discours sensationnaliste de la presse (quasiment hagiographique) et de l'approche documentarisante une telle transcription filmique du discours des adolescents. Puisqu'au fil de la séquence, l'arme semble effectivement "sortir du jeu", passant d'écran en écran, avant d'apparaitre réellement dans le salon d'Eric.

Les jeux vidéo occupent en définitive une place particulière au sein d'Elephant. Dans un premier temps, le traitement qui leur est réservé contraste avec les stratégies globales du film. Plus encore: par un jeu d'hybridité, la convocation de codes vidéoludiques permet au film de déployer son esthétique, tout en rejetant les jeux hors du regard «neutre» et dépassionné qu'ils contribuent à instaurer. Dans un second temps, à travers la séquence qui les thématise, s'immisce un discours qui rappelle l'atmosphère alarmiste et réductrice qui dominait les débats sur les jeux vidéo il y a un peu plus d'une dizaine d'années. A défaut d'être un gamer
44 Amy Harmon parle alors d'internet comme "medium refuge" pour les jeunes gens ostracisés au lycée puis poursuit: "M. Harris passait également du temps en ligne où il gérait son propre site internet et jouait au jeu vidéo Quake [successeur de Doom, développé pa le même studio], dans lequel le joueur traque et tue ses adversaires" (Amy Harmon, Terror in Littelton: The Outcasts; Theme Song on the Internet: The Pain of Social Ostracism, numéro spécial "The Columbine Tapes", Times, 24 avril 1999; notre traduction).

$\mathbf{4 5}$ Cet écart entre le jeu fictif d'Elephant et les jeux de tir est mentionné dans le dossier pédagogique produit par le Ministère français de l'éducation nationale. Pour l'auteur, il s'agit d'un choix visant à une neutralité dans la représentation des victimes, qui corrobore à la caractérisation d'Eric et Alex. L'analyse est cependant tronquée par une volonté de distinguer les jeunes tueurs des "gamers" normaux: "Ce qui différencie Alex et Eric des autres 'gamer, c'est qu'ils passent à l'acte en transposant le jeu dans leur réalité. Ils n'ont plus (ou pas) les notions judéo-chrétiennes de Bien et de Mal". Ludovic Graillat, Jeux video: une culture de la violence. Texte présenté dans le dossier pédagogique réalisé par le Ministère français de l'éducation nationale.

46 La coupe de la vue subjective sur le jeu fictif à Alex jouant du piano intervient après que le "joueur" a changé d'arme; divulguant ainsi une modalité de l'interface supplémentaire et faisant écho par cet étalage des possibilités paradigmatiques à la "gamme de choix" du site de vente d'arme.

47 Notamment cité dans Nancy Gibbs et Timothy Roche, "The Columbine Tapes", Times, 24 avril 1999. Disponible en ligne: www.time.com/ time/magazine/article/0,9171,992873,00.html [dernière consultation au 10.07.2011]. 
48 Voir toutefois sa passion pour Tomb Rider, qu'il évoque dans un entretien cité dans l'article d'Alain Boillat (référence dans la note 21 de son article). chevronné48, Gus Van Sant joue avec les jeux vidéo dans Elephant (de même dans Gerry, qui annonce la mise en cadre et le traitement des déplacements d'Elephant), et propose un objet inédit d'intermédialité. Que cette résultante découle d'un choix d'auteur ou qu'elle soit le produit d'une conjoncture épistémique nous paraît relativement anecdotique. Cependant, la tension dans l'emploi et l'illustration des jeux vidéo nous ferait penser que la deuxième piste est plus recevable. En marge du cinéma transludique, Elephant permet d'éclairer certaines dynamiques vidéoludiques en les sabotant au profit d'une esthétique contemplative. Les traits qui rapprochent le film des jeux vidéo (personnages-pivots, temporalité algorithmique, etc.) l'éloignent du cinéma dominant pour en faire un objet singulier. Le film atteste aussi d'un regard sociétal sur les jeux vidéo propre à la fin du siècle passé (regard alors dominant) et constitue ainsi un double jalon, à la fois comme trace de l'imaginaire vidéoludique imbriqué dans un objet filmique et produit d'une hybridité multimédiatique. 